Pacific

Journal of

Mathematics

HIGHER DIMENSIONAL LINKS IN A SIMPLICIAL COMPLEX EMBEDDED IN A SPHERE

Kouki TANIYAma 


\title{
HIGHER DIMENSIONAL LINKS IN A SIMPLICIAL COMPLEX EMBEDDED IN A SPHERE
}

\author{
KOUKI TANiYAma
}

We show that any embedding of the $n$-skeleton of a $(2 n+$ $3)$-dimensional simplex into the $(2 n+1)$-dimensional sphere contains a nonsplittable link of two $n$-dimensional spheres.

\section{Introduction.}

Throughout this paper we work in the piecewise linear category. Conway and Gordon showed in [1] that any embedding of the complete graph over six vertices into the 3 -space contains a pair of nontrivially linked circles. We refer the reader to $[\mathbf{6}],[\mathbf{2}],[\mathbf{4}],[\mathbf{3}]$ etc. for related works. In this paper we generalize the result of Conway and Gordon to higher dimensions.

Let $\sigma_{j}^{i}$ be the $i$-skeleton of a $j$-dimensional simplex $\sigma_{j}=\left\langle v_{1}, v_{2}, \ldots, v_{j+1}\right\rangle$ where $v_{1}, v_{2}, \ldots, v_{j}$ and $v_{j+1}$ are the 0 -simplices of $\sigma_{j}$. Let $S^{k}$ be the $k$ dimensional unit sphere. Let $X$ and $Y$ be disjoint $n$-dimensional spheres embedded in $S^{2 n+1}$. Then the linking number $\ell k(X, Y) \in Z$ is defined up to sign, see for example [7]. Then the modulo 2 reduction $\ell k_{2}(X, Y) \in Z / 2 Z$ of $\ell k(X, Y)$ is well-defined. We note that $\ell k_{2}(X, Y) \equiv \ell k_{2}(Y, X)(\bmod 2)$. Let $\mathcal{L}^{n}$ be the set of all unordered pairs of disjoint subcomplices of $\sigma_{2 n+3}^{n}$ each of which is homeomorphic to an $n$-dimensional sphere. We note that each element $(J, K)$ of $\mathcal{L}^{n}$ can be written as

$$
(J, K)=\left(\partial\left\langle v_{a_{1}}, v_{a_{2}}, \ldots, v_{a_{n+2}}\right\rangle, \partial\left\langle v_{b_{1}}, v_{b_{2}}, \ldots, v_{b_{n+2}}\right\rangle\right)
$$

where $\partial$ denotes the boundary and $\left\{a_{1}, a_{2}, \ldots, a_{n+2}\right\} \cup\left\{b_{1}, b_{2}, \ldots, b_{n+2}\right\}=$ $\{1,2, \ldots, 2 n+4\}$. Therefore the number of the elements of $\mathcal{L}^{n}$ is $\left(\begin{array}{l}2 n+4 \\ n+2\end{array}\right) / 2$.

Theorem 1.1. Let $n$ be a non-negative integer. Let $f: \sigma_{2 n+3}^{n} \rightarrow S^{2 n+1}$ be an embedding. Then

$$
\sum_{(J, K) \in \mathcal{L}^{n}} \ell k_{2}(f(J), f(K)) \equiv 1(\bmod 2) .
$$

We note that $\sigma_{5}^{1}$ is the complete graph over six vertices and the case $n=1$ of Theorem 1.1 is what Conway and Gordon actually proved in [1]. By Theorem 1.1 we have that there is at least one $(J, K) \in \mathcal{L}^{n}$ with $\ell k(f(J), f(K)) \equiv 1(\bmod 2)$. Thus we have that any embedding of $\sigma_{2 n+3}^{n}$ into $S^{2 n+1}$ contains a nonsplittable link of two $n$-spheres. 


\section{Proof of Theorem 1.1.}

The idea of the following proof is essentially the same as that of Conway and Gordon in [1].

Lemma 2.1. For any embeddings $f, g: \sigma_{2 n+3}^{n} \rightarrow S^{2 n+1}$,

$$
\sum_{(J, K) \in \mathcal{L}^{n}} \ell k_{2}(f(J), f(K)) \equiv \sum_{(J, K) \in \mathcal{L}^{n}} \ell k_{2}(g(J), g(K))(\bmod 2) .
$$

Proof. Since $n<2 n+1$ we have that both $f$ and $g$ are homotopic to a constant map. Therefore $f$ and $g$ are homotopic. By a standard general position argument we can modify the homotopy between $f$ and $g$ and we may suppose that $f$ and $g$ are connected by a finite sequence of 'crossing changes' of $n$-simplices of $\sigma_{2 n+3}^{n}$. Namely we have a homotopy $H: \sigma_{2 n+3}^{n} \times[0,1] \rightarrow$ $S^{2 n+1} \times[0,1]$ with $H(x, 0)=(f(x), 0), H(x, 1)=(g(x), 1)$ whose multiple points are only finitely many transversal double points of the product of $n$-simplices and $[0,1]$ and no two of them have the same second entry. Then it is enough to show the case that $H$ has just one double point. If the first entries of the preimage of the double point do not lie in disjoint $n$ simplices of $\sigma_{2 n+3}^{n}$ then we have $\ell k_{2}(f(J), f(K)) \equiv \ell k_{2}(g(J), g(K))(\bmod 2)$ for each $(J, K) \in \mathcal{L}^{n}$. Thus we may suppose without loss of generality that the first entries of the preimage lie in $n$-simplices $\left\langle v_{1}, v_{2}, \ldots, v_{n+1}\right\rangle$ and $\left\langle v_{n+2}, v_{n+3}, \ldots, v_{2 n+2}\right\rangle$. Let

$$
\left(J_{1}, K_{1}\right)=\left(\partial\left\langle v_{1}, v_{2}, \ldots, v_{n+1}, v_{2 n+3}\right\rangle, \partial\left\langle v_{n+2}, v_{n+3}, \ldots, v_{2 n+2}, v_{2 n+4}\right\rangle\right)
$$

and

$$
\left(J_{2}, K_{2}\right)=\left(\partial\left\langle v_{1}, v_{2}, \ldots, v_{n+1}, v_{2 n+4}\right\rangle, \partial\left\langle v_{n+2}, v_{n+3}, \ldots, v_{2 n+2}, v_{2 n+3}\right\rangle\right) .
$$

Then we have $\ell k_{2}\left(f\left(J_{i}\right), f\left(K_{i}\right)\right) \equiv \ell k_{2}\left(g\left(J_{i}\right), g\left(K_{i}\right)\right)+1(\bmod 2)$ for $i=1,2$ and $\ell k_{2}(f(J), f(K)) \equiv \ell k_{2}(g(J), g(K))(\bmod 2)$ for $(J, K) \in \mathcal{L}^{n},(J, K) \neq$ $\left(J_{1}, K_{1}\right),\left(J_{2}, K_{2}\right)$ as unordered pair. This completes the proof.

Lemma 2.2. There is an embedding $f: \sigma_{2 n+3}^{n} \rightarrow S^{2 n+1}$ with

$$
\sum_{(J, K) \in \mathcal{L}^{n}} \ell k_{2}(f(J), f(K)) \equiv 1(\bmod 2) .
$$

Proof. We use the fact that $S^{2 n+1}$ is homeomorphic to the join of two $n$ dimensional spheres, see Chapter 1 of [5]. Let $P$ be the join of the two simplicial complices $J_{0}=\partial\left\langle v_{1}, v_{2}, \ldots, v_{n+2}\right\rangle$ and $K_{0}=\partial\left\langle v_{n+3}, v_{n+4}, \ldots, v_{2 n+4}\right\rangle$. Since $\sigma_{2 n+3}=\left\langle v_{1}, v_{2}, \ldots, v_{2 n+4}\right\rangle$ is the join of $\left\langle v_{1}, v_{2}, \ldots, v_{n+2}\right\rangle$ and $\left\langle v_{n+3}\right.$, $\left.v_{n+4}, \ldots, v_{2 n+4}\right\rangle$ we have that $P$ is a subcomplex of $\sigma_{2 n+3}$. Then we have that $\sigma_{2 n+3}^{n}$ is a subcomplex of $P$. Since $P$ is homeomorphic to $S^{2 n+1}$ we have an embedding, say $f$, of $\sigma_{2 n+3}^{n}$ into $S^{2 n+1}$. Let $(J, K) \in \mathcal{L}^{n}$. Then

$$
(J, K)=\left(\partial\left\langle v_{a_{1}}, v_{a_{2}}, \ldots, v_{a_{n+2}}\right\rangle, \partial\left\langle v_{b_{1}}, v_{b_{2}}, \ldots, v_{b_{n+2}}\right\rangle\right)
$$


for some $\left\{a_{1}, a_{2}, \ldots, a_{n+2}\right\}$ and $\left\{b_{1}, b_{2}, \ldots, b_{n+2}\right\}$. If $(J, K) \neq\left(J_{0}, K_{0}\right)$ as unordered pair then we have that the $(n+1)$-simplices $\left\langle v_{a_{1}}, v_{a_{2}}, \ldots, v_{a_{n+2}}\right\rangle$ and $\left\langle v_{b_{1}}, v_{b_{2}}, \ldots, v_{b_{n+2}}\right\rangle$ are contained in $P$. Therefore $f(J)$ and $f(K)$ bound disjoint $(n+1)$-dimensional disks in $S^{2 n+1}$ and we have $\ell k_{2}(f(J), f(K)) \equiv$ $0(\bmod 2)$. It is clear that $\ell k_{2}\left(f\left(J_{0}\right), f\left(K_{0}\right)\right) \equiv 1(\bmod 2)$. This completes the proof.

Theorem 1.1 follows immediately from Lemma 2.1 and Lemma 2.2.

Remark 2.3. If we consider a general position map $f: \sigma_{j+k+3}^{k} \rightarrow S^{j+k+1}$ for $0 \leq j \leq k$ and consider all pair $(J, K)$ of disjoint $j$-sphere and $k$-sphere in $\sigma_{j+k+3}^{k}$, then we have a result that is a generalization of Lemma 2.1. The proof is essentially the same. However it turns out that the sum of $\ell k_{2}$ is zero whenever $j<k$. In fact, for any finite simplicial complex $Q$ and $j<k$, there is a general position map $f: Q \rightarrow S^{j+k+1}$ whose image is contained in the upper hemisphere and whose restriction to the $j$-skeleton of $Q$ is an embedding into the equator $S^{j+k} \subset S^{j+k+1}$. Then it is easy to see that $\ell k_{2}(f(J), f(K))=0$ for any pair $(J, K)$ of disjoint $j$-sphere and $k$-sphere in $Q$.

Acknowledgement. The author would like to thank Professor Toshiki Endo for his helpful comment.

\section{References}

[1] J.H. Conway and C.McA. Gordon, Knots and links in spatial graphs, J. Graph Theory, 7 (1983), 445-453.

[2] T. Kohara and S. Suzuki, Some remarks on knots and links in spatial graphs, Knots 90, ed. A. Kawauchi, Walter de Gruyter, Berlin-New York, (1992), 435-445.

[3] T. Otsuki, Knots and links in certain spatial complete graphs, J. Combin. Theory Ser. B., 68 (1996), 23-35.

[4] N. Robertson, P. Seymour and R. Thomas, Linkless embeddings of graphs in 3-space, Bull. Amer. Math. Soc., 28 (1993), 84-89.

[5] D. Rolfsen, Knots and Links, Math. Lecture Series, 7, Publish or Perish Inc., Berkeley, 1976.

[6] H. Sachs, On spatial analogue of Kuratowski's theorem on planar graphs, Lecuture Notes in Math., 1018, Springer-Verlag, Berlin-Heidelberg, (1983), 230-241.

[7] H. Seifert and W. Threlfall, A Textbook of Topology, Pure and Applied Math., 89, Academic Press, New York-London-Toronto-Sidney-San Francisco, 1980.

Received October 6, 1998 and revised February 23, 1999.

Department of Mathematics

TOKYO Woman's Christian University

Zempukuji 2-6-1, Suginamiku

TOKYO, 167-8585

JAPAN 
E-mail address: taniyama@twcu.ac.jp 